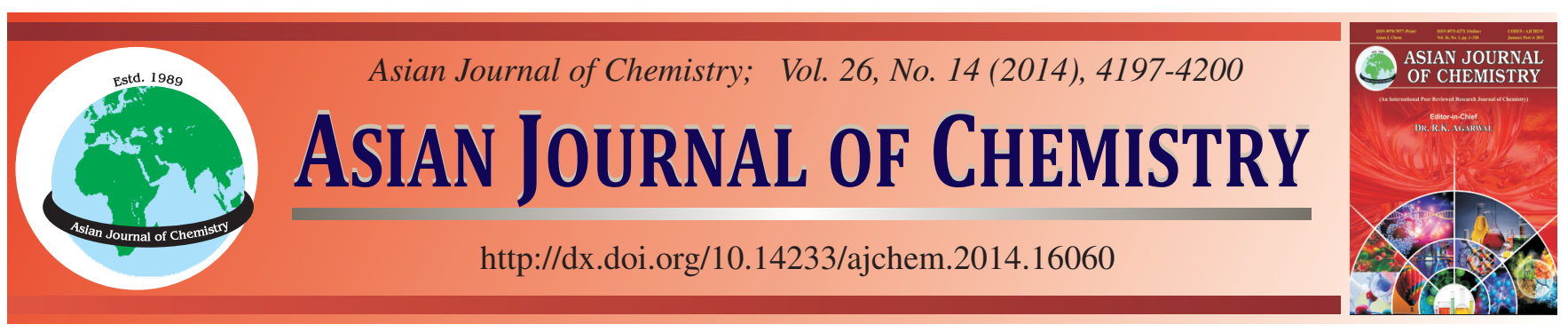

\title{
Adsorption of Isonicotinamide on Loughlinite (Natural Na-Sepiolite): An FT-IR Spectroscopic Study
}

\author{
Sevim Akyuz and TANil Akyuz
}

Istanbul Kultur University, Science and Letters Faculty, Physics Department, Atakoy Campus, 34156 Bakirkoy, Istanbul, Turkey

*Corresponding author: Fax: +90 212 4658318; Tel: +90 212 4984401; E-mail: s.akyuz@iku.edu.tr

Received: 18 July 2013;

Accepted: 24 September 2013;

Published online: 5 July 2014;

AJC-15440

\begin{abstract}
In this study adsorption of isonicotinamide on loughlinite (Na-sepiolite) has been investigated by FT-IR spectroscopy. The adsorption of isonicotinamide showed a considerable alteration on both $\mathrm{Si}-\mathrm{OH}$ stretching and deformation peaks of loughlinite structure. Spectroscopic results indicate that adsorbed isonicotinamide molecules onto loughlinite are coordinated to surface silanol groups and/or Lewis acidic centers by H-bonding interaction through pyridine ring nitrogen lone pairs. The amino nitrogen is found to be relatively in active to adsorbing sites.
\end{abstract}

Keywords: Adsorption, Clays, IR spectroscopy, Isonicotinamide, Loughlinite, Sepiolite.

\section{INTRODUCTION}

Compounds containing pyridine rings are distributed over a wide space in nature and have several important applications. Isonicotinamide (pyridine-4-carboxamide, $\mathrm{C}_{6} \mathrm{H}_{6} \mathrm{~N}_{2} \mathrm{O}$ ) is a pyridine derivative with amide group in the 4-position and has a pharmaceutical importance due to its antitubercular, antipyretic and antibacterial properties ${ }^{1-3}$. Furthermore, mixed salts of this molecule find extensive use in drug industry ${ }^{4}$.

Loughlinite, which is known as natural $\mathrm{Na}$-sepiolite, belongs to palygorskite-sepiolite group of phyllosilicates class of clay minerals ${ }^{5,6}$. Sepiolite and loughlinite have a fibrous like morphology with a distinctive layered appearance ${ }^{5-8}$. Loughlinite ${ }^{5}$ has the structural formula $\mathrm{Na}_{2} \mathrm{Mg}_{3} \mathrm{Si}_{6} \mathrm{O}_{16} \cdot 8 \mathrm{H}_{2} \mathrm{O}$ and sepiolite ${ }^{7}$ the formula $\mathrm{Mg}_{4} \mathrm{Si}_{6} \mathrm{O}_{15}(\mathrm{OH})_{2} \cdot 6 \mathrm{H}_{2} \mathrm{O}$. Both sepiolite and loughlinite are chain minerals. Sepiolite is structurally formed by an alteration of blocks and channels that grow up in the fibre direction $(c \text {-axis })^{8}$. Each structural block is build up of two tetrahedral silica sheets enclosing a central octahedral magnesia sheet in a similar way that occurs in other 2:1 silicates such as talc, but in sepiolite there are discontinuities and inversions of the silica sheet that give rise to those structural channels $^{8}$. Loughlinite is virtually identical in appearance to sepiolite both in a hand specimen and microscopically. However, field observations and mineralogical determinations indicate that loughlinite and sepiolite are formed authigenically and independently in different physicochemical environments rather than being the product of transformation of one to the other ${ }^{6}$.
In our previous studies, we were investigated the interaction of isonicotinamide with montmorillonite and saponite 9 . Continuing our studies on the adsorption of drugs on the clay surfaces $^{9-12}$, in this study the sorption of 4-pyridinecarboxamide (isonicotinamide) onto natural Na-sepiolite (loughlinite) from Anatolia has been investigated using FT-IR spectrometry. Up to our best of knowledge no study has been reported on the adsorption of isonicotinamide onto loughlinite. The objective of this paper is to examine the generated surface species on the clay and to investigate the interaction between isonicotinamide and loughlinite framework.

EXPERIMENTAL
In the present work, loughlinite from Mihalliccik-
Eskisehir region of Anatolia (Turkey) was employed. The clay
sample was ground to fine powder of $>0.5 \mathrm{~mm}$ particle size
and was analysed by X-ray diffraction, differential thermal
and IR spectroscopic techniques for purity. The results indicate
that loughlinite is associated with analcime (approximately
$8 \%$ ) and $\mathrm{Al}_{2} \mathrm{O}_{3}($ approximately $6 \%$ ), and has detectable calcite
impurity (IR; $1435 \mathrm{~cm}^{-1}$ ). The chemical composition of lough-
linite was given in previous study ${ }^{10}$. Isonicotinamide used was
reagent grade (Fluka) and used as received. Isonicotinamide
treated loughlinite was prepared by immersing clay in either
aqueous solution of the organic molecule in sealed bottle at
room temperature for 2 d or by solid-solid reactions between
clay and the organic molecule, analogous to that described by
Ogawa et al.$^{13}$ and Khaorapapong et al.$^{14}$. In the former case
clay organic suspension was centrifuged at $7000 \mathrm{~g}$ for 10 min.


IR spectra of the clay organic complexes were recorded on a Bruker Tensor spectrometer in the $4000-400 \mathrm{~cm}^{-1}$ region $\left(0.5 \mathrm{~cm}^{-1}\right.$ resolution) by using $\mathrm{KBr}$ pellet technique.

\section{RESULTS AND DISCUSSION}

The molecular model of isonicotinamide is given in Fig. 1. FT-IR spectra of solid isonicotinamide, starting loughlinite and isonicotinamide treated loughlinite are given in Fig. 2. In the $2700-2600 \mathrm{~cm}^{-1}$ region of the IR spectra of isonicotinamide treated loughlinite, we did not observe any band which could be attributed to the $\mathrm{N}^{+} \mathrm{H}$ stretching vibration, indicating that cationic surface species were not generated on loughlinite.

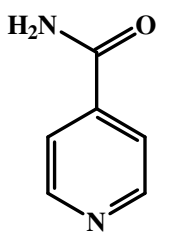

Fig. 1. Molecular model of isonicotinamide

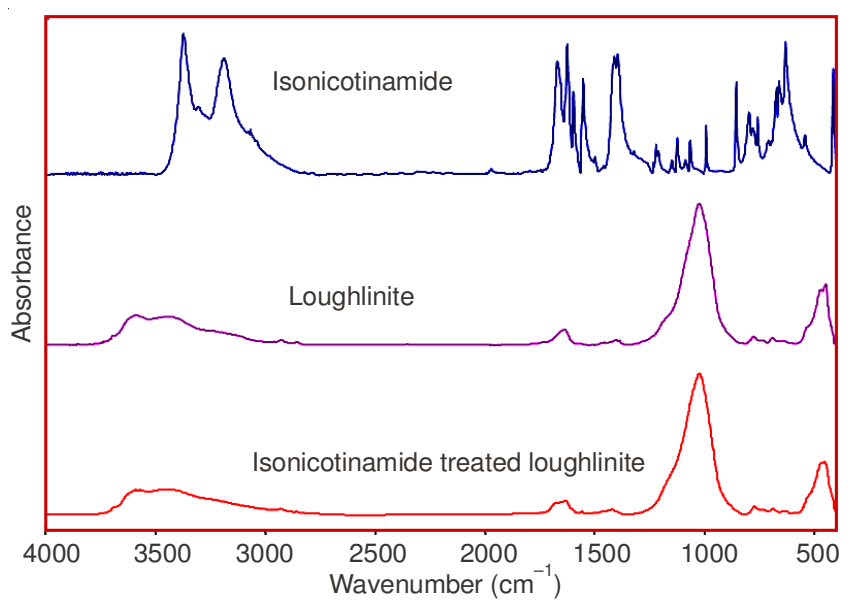

Fig. 2. FT-IR spectra of solid isonicotinamide (top), loughlinite (middle) and isonicotinamide treated loughlinite (bottom)

The possible binding sides of isonicotinamide are the ring nitrogen, the amino nitrogen and/or the carbonyl oxygen. It is well known that the adsorption of the organic molecule onto clay surface gives rise to the changes in the IR spectra of the interacting species. In order to determine the interaction mechanism of adsorbed isonicotinamide molecules by loughlinite, the vibrational wavenumbers of adsorbed molecules are carefully investigated, by taking into account the coordination effects through the ring, through the $\mathrm{NH}_{2}$ group and through the $>\mathrm{C}=\mathrm{O}$ group. If the coordination occurs through the amino nitrogen atom, it is expected that a great reduction would take place in the $\mathrm{NH}_{2}$ and the $\mathrm{C}-\mathrm{NH}_{2}$ stretching wavenumbers ${ }^{15}$ due to the perturbation of the amino group by coordination. For example in the case of aniline, benzidine or $p$-phenylenediamine complexes, the values of the $\mathrm{NH}_{2}$ and the $\mathrm{C}-\mathrm{NH}_{2}$ stretching modes were found at lower values than the corresponding ones in the free molecule and the red shifts were 150$220 \mathrm{~cm}^{-1}$ for $\mathrm{NH}_{2}$ stretching and $50-60 \mathrm{~cm}^{-1}$ for $\mathrm{C}-\mathrm{NH}_{2}$ stretching vibrations ${ }^{15,16}$. On the other hand, it was known that the ring stretching vibrations around $1600-1400 \mathrm{~cm}^{-1}$ and the ringbreathing mode increase in wavenumber upon $\mathrm{H}$ bond formation or adsorption at Lewis acid sites ${ }^{17}$. In order to investigate clay-organic interaction, we compared our results with those of solid isonicotinamide, isonicotinamide isolated in cryogenic inert matrixes ${ }^{18}$ and $\mathrm{Ni}$ (isonicotinamide) $)_{2} \mathrm{Ni}(\mathrm{CN})_{4}$ complex, in which the coordination of the isonicotinamide with the metal occurs through the pyridine nitrogen ${ }^{19}$. The vibrational wavenumbers of adsorbed isonicotinamide are tabulated in Table-1, in comparison to those of solid isonicotinamide, isonicotinamide in Xe matrix and isonicotinamide complex.

Although the $\mathrm{NH}_{2}$ stretching vibrations are obscured by strong $\mathrm{OH}$ stretching vibrations of the clay framework, the $\mathrm{C}-\mathrm{NH}_{2}$ stretching mode is clearly observed in IR spectra of isonicotinamide treated loughlinite. The $\mathrm{C}-\mathrm{NH}_{2}$ stretching vibration is observed at $1391 \mathrm{~cm}^{-1}$ in the IR spectra of solid isonicotinamide as a strong band ${ }^{19}$. The $1800-1300 \mathrm{~cm}^{-1}$ region of the FTIR spectra of starting loughlinite and isonicotinamide treated loughlinite are given in Fig. 3 comparatively. The FTIR spectrum of isonicotinamide treated loughlinite, after subtraction of louglinite bands is given in Fig. 4 in comparison to that of solid isonicotinamide. The strong band observed at $1395 \mathrm{~cm}^{-1}$ in the IR spectra of solid isonicotinamide is attributable to $\mathrm{C}-\mathrm{NH}_{2}$ stretching mode (Fig. 4). The corresponding mode of the adsorbed isonicotinamide is assigned to $1420 \mathrm{~cm}^{-1}$ medium band, observed in the IR spectrum of isonicotinamide treated loughlinite (Fig. 3). This mode is clearly seen in the subtracted IR spectrum of clay-organic complex (isonicotinamide treated loughlinite-loughlinite), shown in Fig. 4. Therefore, the investigation of the vibrational spectra of isonicotinamide

\begin{tabular}{|c|c|c|c|c|}
\hline \multicolumn{5}{|c|}{$\begin{array}{c}\text { TABLE-1 } \\
\text { WAVENUMBERS }\left(\mathrm{cm}^{-1}\right) \text { OF ADSORBED ISONICOTINAMIDE (INA) }\end{array}$} \\
\hline Assignment & INA solid & INA in Xe matrix $(20 \mathrm{~K})^{\mathrm{a}}$ & INA complex $^{b}$ & INA on loughlinite \\
\hline$v(\mathrm{C}=\mathrm{O})$ & 1668 & $1711-1708$ & 1682 & $1693-1681$ \\
\hline$\delta\left(\mathrm{NH}_{2}\right)$ & 1624 & $1578-1571$ & 1629 & 1625 \\
\hline$v_{\text {ring }}$ & 1596 & 1586 & 1617 & 1601 \\
\hline$v_{\text {ring }}$ & 1552 & 1559 & 1551 & 1556 \\
\hline$v_{\text {ring }}$ & 1496 & $1507^{c}$ & 1504 & $1505-1498$ \\
\hline$\delta \mathrm{CH}$ & 1410 & 1407 & 1418 & 1439 \\
\hline $\mathrm{v}(\mathrm{CN})_{\text {amide }}$ & 1395 & 1360 & 1400 & 1420 \\
\hline$v_{\text {ring }}$ & 994 & 993 & 1030 & 1004 \\
\hline$\gamma(\mathrm{C}-\mathrm{C})$ & 757 & 748 & 761 & 759 \\
\hline$\gamma_{\text {ring }}$ & 412 & 418 & 419 & 420 \\
\hline
\end{tabular}




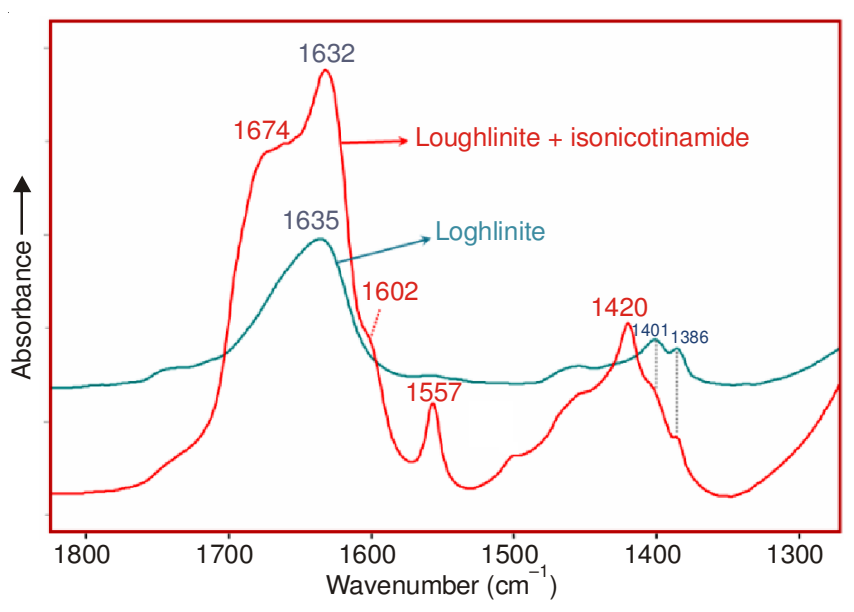

Fig. 3. 1800-1300 $\mathrm{cm}^{-1}$ region of the FT-IR spectra of loughlinite before and after treatment with isonicotinamide

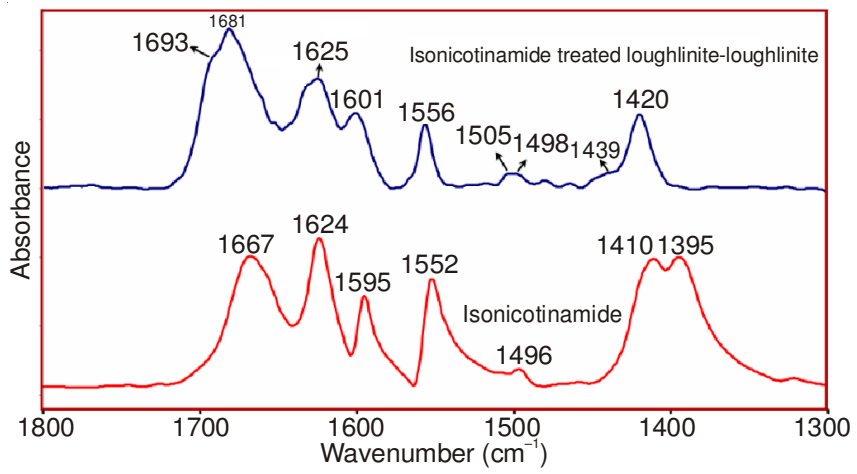

Fig. 4. The 1800-1300 $\mathrm{cm}^{-1}$ region of the FT-IR spectra of solid isonicotinamide (bottom) and isonicotinamide treated loughlinite after subtraction of loughlinite bands

treated loughlinite indicates that no reduction in frequency on the $\mathrm{CN}$ vibrational mode of adsorbed molecule in comparison to that of solid phase is present. Contrarily this vibrational mode is found to be higher in wavenumber than those solid isonicotinamide. The $\mathrm{C}-\mathrm{NH}_{2}$ stretching mode was observed at $1400 \mathrm{~cm}^{-1}$ in the IR spectrum of $\mathrm{Ni}$ (isonicotinamide) ${ }_{2} \mathrm{Ni}(\mathrm{CN})_{4}$ complex ${ }^{19}$, in which the coordinatin occurs through the ring nitrogen. The increase in frequency of the $\mathrm{C}-\mathrm{NH}_{2}$ stretching mode upon adsorption of isonicotinamide indicates the increase of pi character of the $\mathrm{C}-\mathrm{N}\left(\mathrm{NH}_{2}\right)$ bond. Thus, the spectral results suggest that the amino nitrogen of isonicotinamide is not involved in the coordination.

In a recent study ${ }^{17}$, the adsorption of 2-chloropyridine on oxide surfaces were investigated and the vibrational wavenumber shifts that accompany the different types of interaction of the aromatic molecule with surface sites were calculated. It was shown that the ring stretching vibrations around 1600$1400 \mathrm{~cm}^{-1}$, increase in wavenumber upon $\mathrm{H}$ bond formation or adsorption at Lewis acid sites ${ }^{17}$. In our previous study the coordination through the ring nitrogen effects on isonicotinamide $^{20}$ and isoniazid ${ }^{21}$ vibrational wavenumbers were calculated using a model compound of INA- $\mathrm{Al}(\mathrm{OH})_{3}$ complexes (INA = isonicotinamide or isoniazid) and the results were in agreement to the previous findings ${ }^{17}$. It was found that in addition to the pyridine ring and $\mathrm{CH}$ modes around 1600-1400 $\mathrm{cm}^{-1}$, ring breathing mode is also sensitive to coordination through pyridine ring to nitrogen. The ring mode around 1000 $\mathrm{cm}^{-1}$ is obscured by strong $\mathrm{Si}-\mathrm{O}$ stretching mode of loughlinite. But The second derivative profiles of the absorbtion spectra give valuable information about the positions of the overlapping bands (Fig. 5). Comparison of the second derivative profiles of starting loughlinite and isonicotinamide treated loughlinite indicates an additional peak at $1004 \mathrm{~cm}^{-1}$ in the latter case, which is not present in the former, that can be attributed to the ring breathing mode of adsorbed isonicotinamide. Table-1 showed that the vibrational wavenumbers of adsorbed isonicotinamide are found to be more close to those of isonicotinamide coordinated through ring nitrogen, than free isonicotinamide. The results indicate that adsorbed isonicotinamide molecules are coordinated through the ring nitrogen lone pairs.

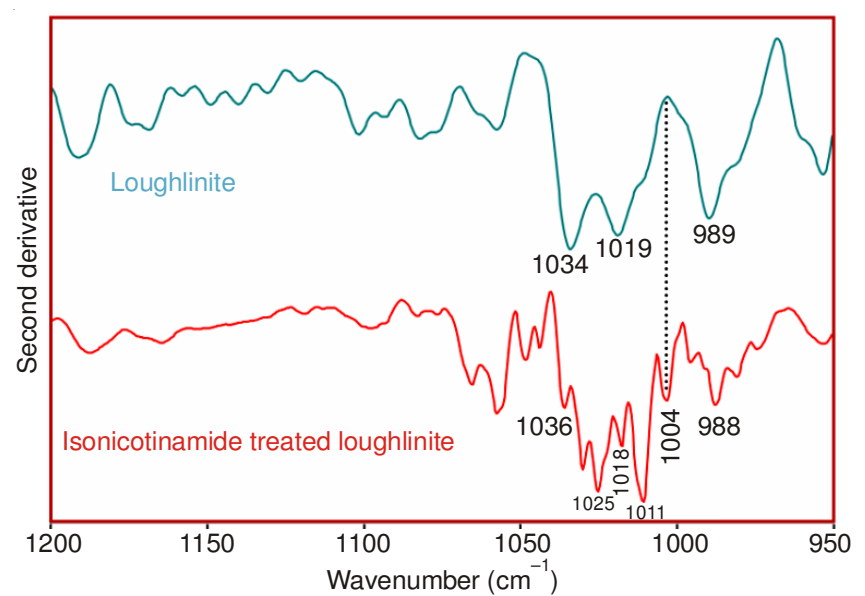

Fig. 5. The second derivative profiles of the absorption IR spectra of starting loughlinite (top) and isonicotinamide treated loughlinite (bottom)

The most probable binding sites of sepiolite group of clays $^{8}$ are expected to be surface hydroxyls and Lewis acidic centers. It is well known that in this group of clays, the broken $\mathrm{Si}-\mathrm{O}-\mathrm{Si}$ bonds of the terminal silica tetrahedra on the external surfaces of compensate their residual charge by accepting a proton or hydroxyl group and form $\mathrm{Si}-\mathrm{OH}$ groups ${ }^{8}$. The $\mathrm{Si}-\mathrm{OH}$ stretching and the deformation vibrations are expected around 3700 and $800 \mathrm{~cm}^{-1}$, respectively ${ }^{22}$. Alterations of these bands indicate interactions of the adsorbate with the silanol groups. The $\mathrm{OH}$ stretching region of isonicotinamide treated loughlinite is given in Fig. 6. As seen in Fig. 6, the intensity of Si-OH vibration is found to increase and shift to lower wavenumber upon adsorption of isonicotinamide. This mode is observed at 3693 and $3683 \mathrm{~cm}^{-1}$ in the IR spectra of loughlinite and isonicotinamide treated loughlinite, respectively. We also observed the alterations of the $\mathrm{Si}-\mathrm{OH}$ deformation bands around $800 \mathrm{~cm}^{-1}$. The Si-OH deformation bands of loughlinite before and after treatrment with isonicotinamide is shown in Fig. 7, comparatively. The center of the band profile of the $\mathrm{Si}-\mathrm{OH}$ deformation region is found to shift slightly to lower wavenumber upon adsorption of isonicotinamide. In order to see the alterations of the Si-OH deformation band, we performed band component analysis. The band component analyses of the Si-OH deformation bands of isonicotinamide treated loughlinite are given in Fig. 8. The two main components of the $\mathrm{Si}-\mathrm{OH}$ deformation bands are observed at 778 and $769 \mathrm{~cm}^{-1}$ in 


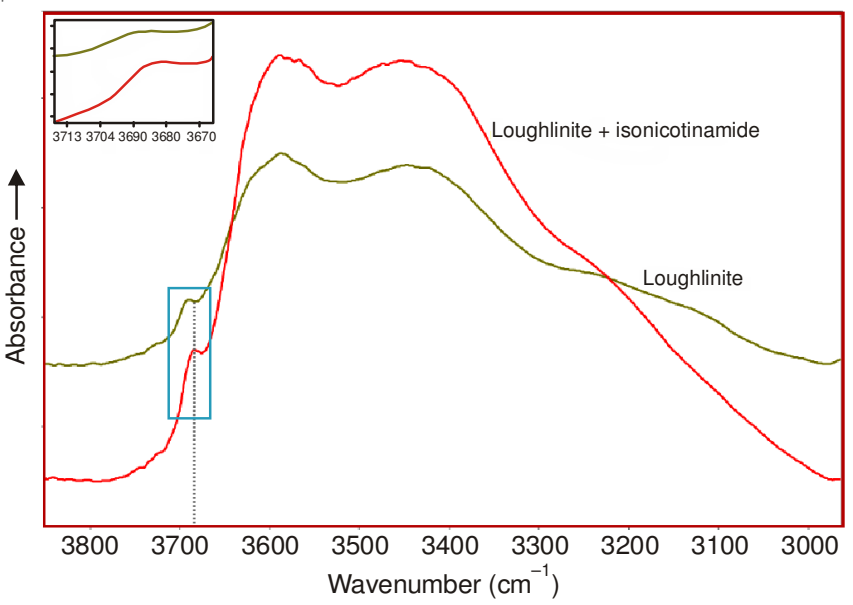

Fig. 6. $\quad 3800-3000 \mathrm{~cm}^{-1}$ region of the FT-IR spectra of natural loughlinite and isonicotinamide treated loughlinite. The $3745-3675 \mathrm{~cm}^{-1}$ regions of both samples is shown comparatively in the small frame given above

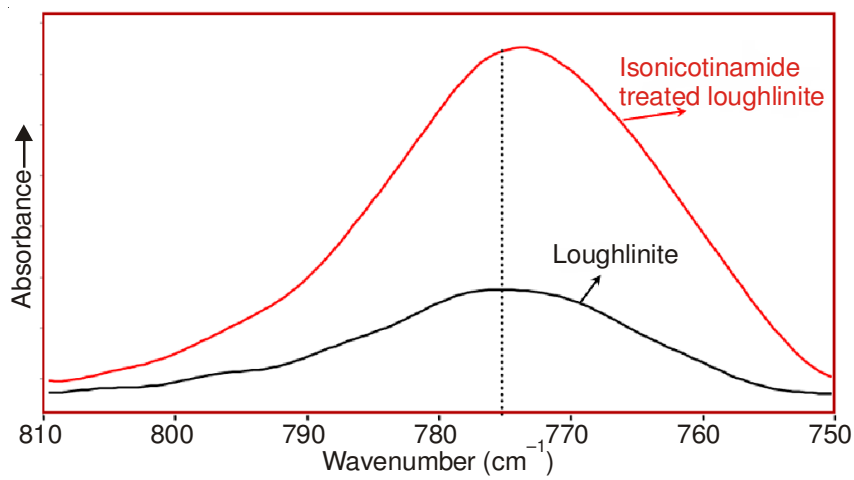

Fig. 7. Si-OH deformation band of loughlinite and isonicotinamide treated loughlinite
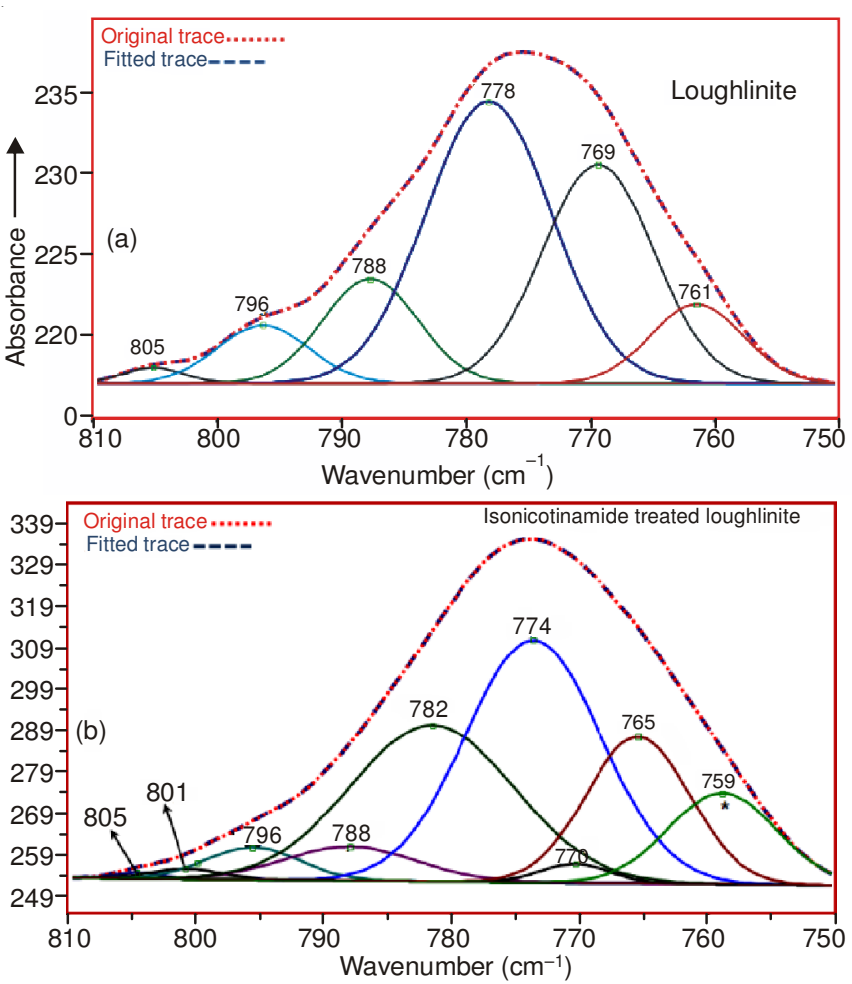

Fig. 8. The band component analysis of the $810-750 \mathrm{~cm}^{-1}$ region of the FT-IR spectra of loughlinite (a) and isonicotinamide-treated loughlinite (b). *Isonicotinamide band the IR spectrum of pure loughlinite, corresponding components are observed at 774 and $765 \mathrm{~cm}^{-1}$, in that of isonicotinamide treated loughlinite. If adsorbed isonicotinamide molecule interacts with surface silanol groups, alterations in the $\mathrm{Si}-\mathrm{OH}$ stretching and deformation bands are expected. That is what we observed. Thus, the spectroscopic results indicate that isonicotinamide molecules adsorbed on loughlinite are mostly coordinated to surface hydroxyls by H-bonding interaction. Direct or indirect (though the water bridges) coordination to Lewis acidic centers can not be excluded.

\section{Conclusion}

The IR spectroscopic investigations are found to be very informative in shedding light on the nature of the surface species formed on clays. Based on the spectroscopic investigations, we propose isonicotinamide molecules adsorbed on loughlinite are coordinated to surface hydroxyls and/or Lewis acidic centers by $\mathrm{H}$-bonding interaction through pyridine ring nitrogen lone pairs.

\section{ACKNOWLEDGEMENTS}

This study is supported by the Research Fund of Istanbul Kultur University

\section{REFERENCES}

1. R.A. Olsen, L. Liu, N. Ghaderi, A. Johns, M.E. Hatcher and L.J. Mueller, J. Am. Chem. Soc., 125, 10125 (2003).

2. R.K. Murray, D.K. Granner, P.A. Mayes and V.W. Rodwell, Harper's Biochemistry, Prentice Hall International Inc., London, edn 22, p. 332 (1990).

3. S. Yurdakul, A. Ataç, E. Sahin and S. Ide, Vib. Spectrosc., 31, 41 (2003).

4. J.L. Castro, J.F. Arenas, M.R. Lopez-Ramirez, J. Soto and J.C. Otero, J. Colloid Interf. Sci., 396, 95 (2013).

5. J.J. Fahey, M. Ross and J.M. Axelrod, Am. Mineral., 45, 270 (1960).

6. S. Kadir, H. Bas and Z. Karakas, Can. Mineral., 40, 1091 (2002).

7. K. Brauner and A. Preisinger, Tschermaks Mineral. Petrogr. Mitt., 6, 120 (1956).

8. J.M. Serratosa, In Proceedings of International Clay Conference, 1978, edited by M.M. Mortland and V.C. Farmer, Elsevier, Amsterdam, p. 99 (1979).

9. S. Akyuz and T. Akyuz, Vib. Spectrosc., 42, 387 (2006).

10. S. Akyuz and T. Akyuz, Asian J. Chem., 20, 3767 (2008).

11. S. Akyuz and T. Akyuz, Asian J. Chem., 22, 546 (2010).

12. T. Akyuz and S. Akyuz, Asian J. Chem., 23, 3211 (2011).

13. M. Ogawa, T. Hashizume, K. Kuroda and C. Kato, Inorg. Chem., 30, 584 (1991).

14. N. Khaorapapong, K. Kuroda, T. Hashizume and M. Ogawa, Appl. Clay Sci., 19, 69 (2001).

15. E. Akalin and S. Akyuz, J. Mol. Struct., 482-483, 175 (1999).

16. E. Akalin and S. Akyuz, J. Mol. Struct., 565-564, 579 (2001).

17. T.J. Dines, L.D. MacGregor and C.H. Rochester, Spectrochim. Acta A, 59, 3205 (2003).

18. A. Borba, A. Gómez-Zavaglia and R. Fausto, J. Phys. Chem., 112, 45 (2008).

19. S. Yurdakul and A. Atac, Spectrosc. Lett., 37, 33 (2004).

20. E. Akalin, A. Yilmaz and S. Akyuz, J. Mol. Struct., 744-747, 881 (2005).

21. E. Akalin, S. Akyuz and T. Akyuz, Asian J. Chem., 22, 4111 (2010).

22. S. Akyuz and T. Akyuz, J. Mol. Struct., 744-747, 47 (2005). 